\title{
Breaking Friedel's Law in Polar Two Dimensional Materials
}

\author{
Pratiti Deb ${ }^{1}$, Yimo Han ${ }^{1}$, Saien Xie ${ }^{1}$, Megan E. Holtz ${ }^{1}$, Jiwoong Park ${ }^{2,3}$ and David A. Muller ${ }^{1,4}$ \\ 1. School of Applied and Engineering Physics, Cornell University, Ithaca, USA. \\ 2. Department of Chemistry, Cornell University, Ithaca, USA. \\ 3. Department of Chemistry, University of Chicago, Chicago, USA. \\ 4. Kavli Institute at Cornell for Nanoscale Science, Ithaca, USA.
}

Recent advances in direct-electron detectors for electron diffraction have provided new opportunities for studying localized phenomena in crystal structures. Here, we extract fully quantitative diffraction information from atomically thin two dimensional (2D) materials using a high-speed, high dynamic range detector [1], and explore how Friedel's law is broken in monolayer 2D materials. Friedel's Law states that a diffraction pattern will have equal intensities for both the $h k l$ and $\overline{h k l}$ points, i.e. $\mathrm{I}(h k l)=$ $\mathrm{I}(\overline{h k l})$, as expected for kinematic scattering from a real potential, even for a polar material. Historically, anomalous scattering that reflects the polarity, i.e. breaking of in-plane inversion symmetry, was attributed to multiple scattering from different atoms in a thick crystal, each of which was assumed to be a weak scatterer [2]. Here, we show from both calculation and experiment that for a polar 2D material, even a monolayer acts as a sufficiently strong phase object to breaks Friedel's law, allowing us to map the polarity of the crystal across 5 orders of magnitude in length scale.

In previous dark field transmission electron microscopy (DF-TEM) of monolayer molybdenum disulfide $\left(\mathrm{MoS}_{2}\right)$ [3], an anomalous contrast was observed between the $h k l$ and $\overline{h k l}$ spots in the diffraction pattern. Experiments using our newly developed high dynamic range electron microscope pixel array detector (EMPAD), which acquires a diffraction pattern in $0.86 \mathrm{~ms}$ at each scan position of the electron beam, enabled us to numerically quantify the extent to which Friedel's law is broken in tungsten disulfide $\left(\mathrm{WS}_{2}\right)$ /tungsten diselenide $\left(\mathrm{WSe}_{2}\right.$ ) lateral heterojunctions at $120 \mathrm{keV}$ and provided evidence of the polarity (Fig 1d), in agreement with results from DF-TEM of the same sample (Fig 1a-c). Diffraction mapping experiments using the EMPAD at $60 \mathrm{keV}$ on molybdenum disulfide $\left(\mathrm{MoS}_{2}\right)$ and $\mathrm{WS}_{2}$ monolayers also give quantitative measurements of the polarity in these materials (Fig 2c).

In the Born approximation or the weak phase approximation (WPA), where only linear terms in $\sigma \mathrm{V}$ are retained in the expansion of the scattered electron wave function, where $\sigma$ is the interaction parameter and $\mathrm{V}$ is the atomic potential of the material, there is no anomalous contrast. We show analytically that higher order terms in the power series expansion provide the anomalous contrast between $h k l$ and $\overline{h k l}$ spots in the diffraction pattern for polar materials, and calculate this contrast from both the strong phase approximation (SPA) and its power series expansion. The relative anomalous contrasts for six polar materials (Fig 2c) correlates with $\Delta Z=Z(A)-n \times Z(B)$, where $Z$ is the atomic number and the polar $2 D$ material in question is $\mathrm{AB}_{\mathrm{n}}$. Physically, the higher order terms can be thought of as multiple scattering paths from the same atom, resolving the need for multiple layers. We note most numerical multislice codes appear to lack the numerical precision to calculate the effect, which is sensitive to the treatment of the singularity at the nuclear potential. [4]

References:

[1] M. Tate et al, Microscopy and Microanalysis 22 (2016), p. 237-249. 
[2] S. Miyake et al, Acta Crystallographica 8 (1955), p. 335-342.

[3] A. M. van der Zande et al, Nature Materials 12 (2013), p. 554-561.

[4] Work supported by the Cornell Center for Materials Research, an NSF MRSEC (DMR-1120296).
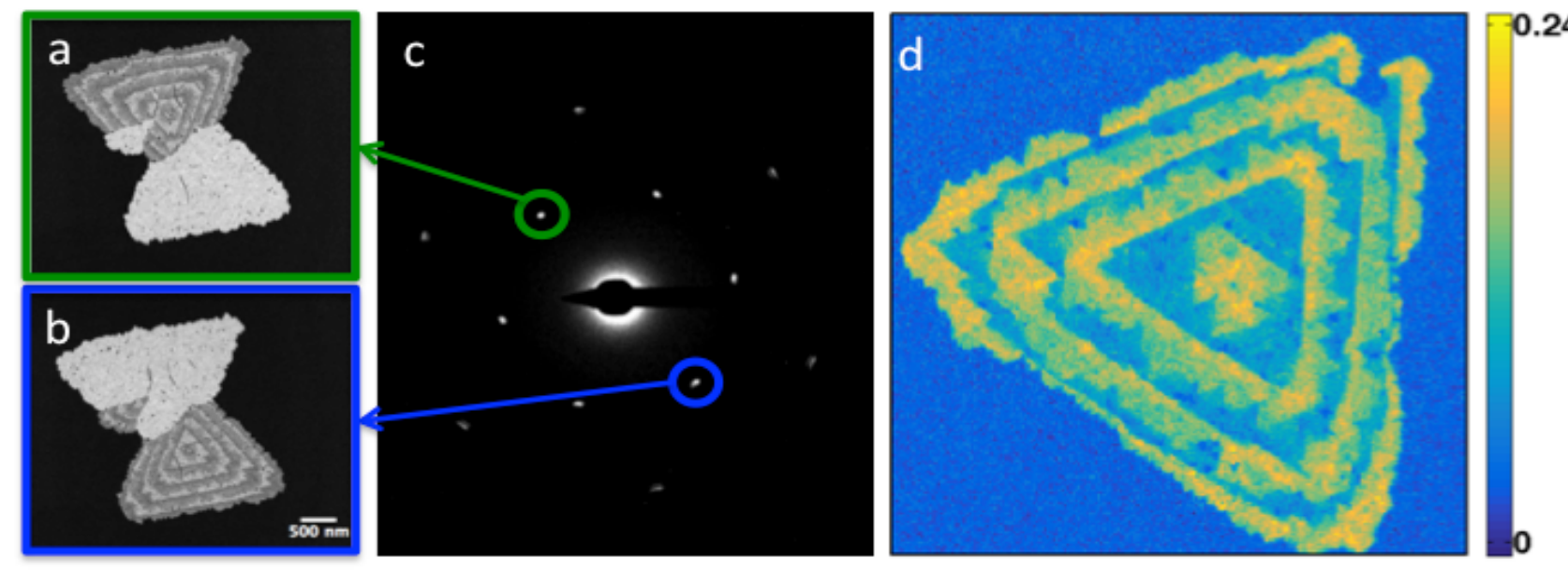

Figure 1. a-b, DF-TEM images from 010 and $0 \overline{10}$ peaks of the diffraction pattern of $\mathrm{WS}_{2} / \mathrm{WSe}_{2}$ lateral heterojunctions demonstrate the polarity of the sample in the inverted brightness of the images for the two peaks. c, Diffraction pattern showing the peaks corresponding to the images in a and b. d, Map of polarity of the sample acquired using electron microscope pixel array detector at beam energy $120 \mathrm{keV}$, where polarity from the anomalous contrast is calculated as $[\mathrm{I}(010)-\mathrm{I}(0 \overline{10})] /[\mathrm{I}(010)+\mathrm{I}(0 \overline{10})]$.
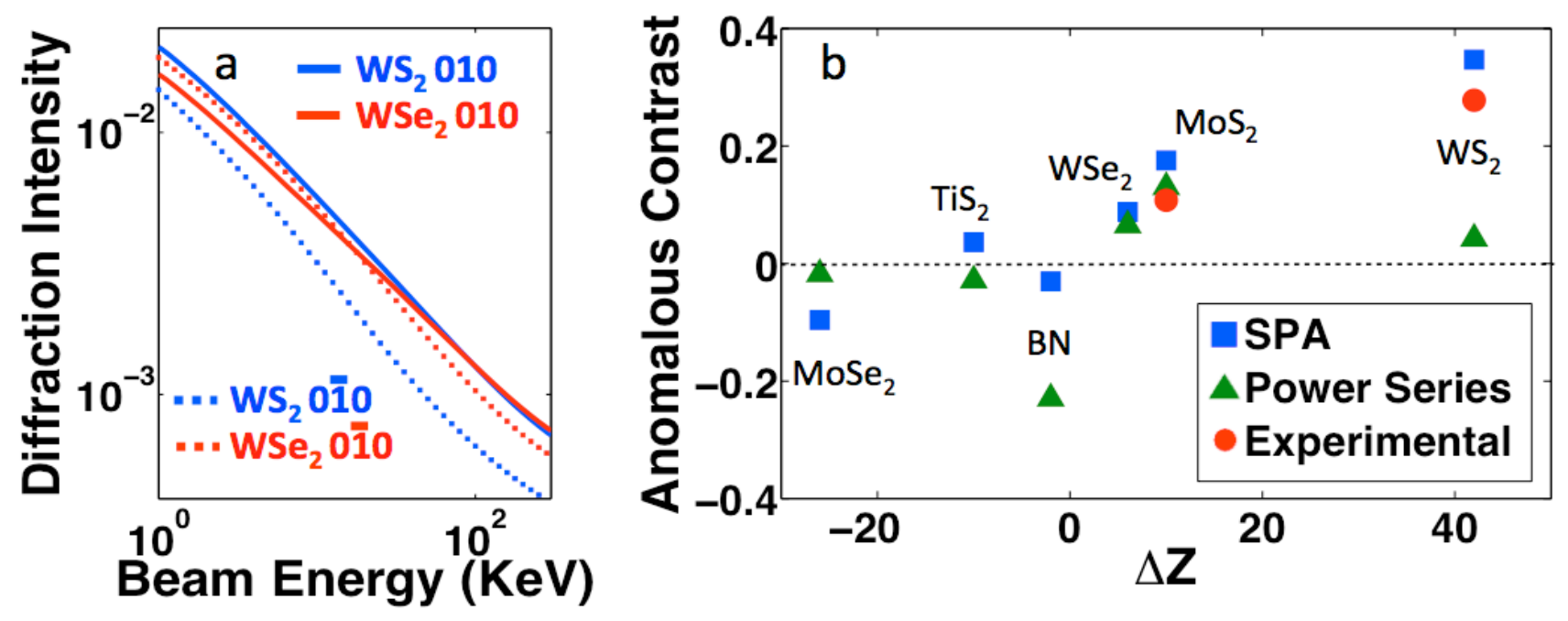

Figure 2. a-b, The normalized diffraction intensity for $\mathrm{WS}_{2}$ and $\mathrm{WSe}_{2}$ is plotted as a function of beam energy for 010 and $0 \overline{10}$ peaks. The difference between the two peaks is higher for $\mathrm{WS}_{2}$ than for $\mathrm{WSe}_{2}$, and the difference between the 010 peaks for the two materials is much lower than the corresponding difference between the $0 \overline{1} 0$ peaks, explaining the difference in contrast between the two triangles in Fig 1a-b. c, The anomalous contrasts calculated from the strong phase approximation (SPA), a power series expansion of the Born wave function, and EMPAD experimental data are plotted as a function of $\Delta \mathrm{Z}$. We see a clear positive correlation between the contrast and $\Delta \mathrm{Z}$ in the SPA, with the experimental data in close agreement. For the power series, convergence and precision issues lead to deviations from the trend for the materials with the heaviest $\left(\mathrm{WS}_{2}\right)$ and lightest $(\mathrm{BN})$ elements respectively. 